\title{
Absolute $\mathrm{Kr}$ 4s-electron photoionization cross sections between 30 and $90 \mathrm{eV}$ measured by photon-induced fluorescence spectroscopy (PIFS)
}

\author{
A. Ehresmann, H. Schmoranzer, F. Vollweiler \\ Fachbereich Physik, Universität Kaiserslautern, 67653 Kaiserslautern, Germany \\ G. Mentzel and K.-H. Schartner \\ I. Physikalisches Institut, Justus-Liebig Universitäl, 35392 Giessen, Germany
}

Received 29 March 1993; revised manuscript received 22 November 1993; accepted for publication 24 November 1993 Communicated by B. Fricke

\begin{abstract}
Abelute $\mathrm{Kr}$ 48-electron photoionization cross sections as a function of the exciting-photon energy between 30 and $90 \mathrm{eV}$ were measured by photon-induced fluorescence spectroscopy (PIFS). The measurements were compared with available experimental data and theoretical calculations.
\end{abstract}

The photoionization of the subvalence s-shells of rare gases is known to be strongly influenced by electron correlations (see, e.g., refs. [1,2], and references therein ). In contrast to the Ar 3s-electron photoionization cross section for which the excitingphoton energy dependence has been intensively investigated during the past five years (see, e.g., ref. [3], and references therein) only a few measurements of the exciting-photon energy dependence of the $\mathrm{Kr}$ 4s-electron photoionization cross section have been published $[2,4-6]$.

Very recently, new theoretical calculations have been carried out $[6,8]$ which underline the necessity of accurate measurements of the $\mathrm{Kr} 48$-electron photoionization cross section in an exciting-photon energy range between threshold and $90 \mathrm{eV}$. None of the measurements $[2,4-6]$ covers this entire region which is of special interest because it contains the minimum of the $\mathrm{Kr}$ 4s-electron photoionization cross section. In the present work these cross sections were determined absolutely by PIFS, extending considerably our preceding measurements [2] which were restricted to the narrow exciting-photon energy region between the 4s-electron ionization threshold and $33.5 \mathrm{eV}$. A brief description of PIFS and the method of evaluating the $\mathrm{Kr}$ 4s-electron photoionization cross sections from the spectroscopic measurements will be given. The results and a comparison with available experimental data and calculations will be presented.

The PIFS method and several of its applications have been previously described in detail [1,9-12]. Thus we mention here only some major differences from the earlier works $[2,12]$ and the important experimental parameters.

Synchrotron radiation from the electron storage ring BESSY, Berlin, was monochromatized by a toroidal-grating primary monochromator (typical bandwidth $0.05 \mathrm{eV}$ at $27.5 \mathrm{eV}$ ) and focussed into a target cell at a typical target gas pressure of $0.01 \mathrm{mbar}$. The primary flux was measured by a Faraday cup. The fluorescence radiation emitted by the excited ions was dispersed by a 1 m-normal incidence monochromator (McPherson 225) and detected by a position-sensitive microchannel plate detector. A typical fluorescence spectrum of $\mathrm{Kr}$ at an exciting-photon energy of $33.0 \mathrm{eV}$ is shown in fig. 1

For the determination of the $\mathrm{Kr}$ 4s-electron photoionization cross section the exciting-photon energy was varied between 30 and $90 \mathrm{eV}$ in steps of differ- 


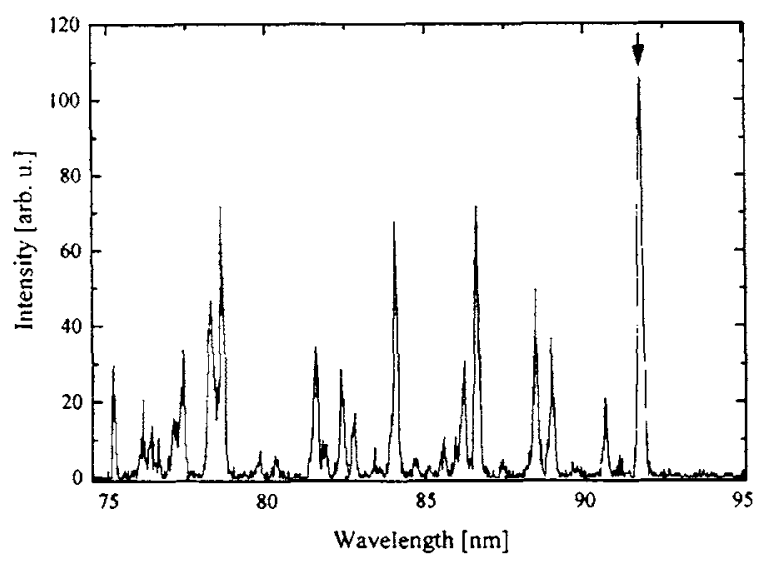

Fig. 1. Typical $\mathrm{Kr}$ fluorescence spectrum between 75 and $95 \mathrm{~nm}$ at an exciting-photon energy of $33.0 \mathrm{eV}$. The $4 s^{1} 4 p^{62} \mathrm{~S}_{1 / 2} \rightarrow$ $4 s^{2} 4 p^{32} P_{3 / 2}$ transition at $91.74 \mathrm{~nm}$ is marked by an arrow. The other lines are satellites.

ent widths and the fluorescence intensity of the two lines arising from the decay of the $\mathrm{Kr} 4 \mathrm{~s}$-hole at 91.74 and $96.49 \mathrm{~nm}$ was recorded as a function of the exciting-photon energy. Then the target gas was changed "in situ" to Ar and the fluorescence intensity from the decay of the Ar 3s-hole at 91.98 and $93.20 \mathrm{~nm}$ was recorded at exactly the same exciting-photon energies. Since the relative quantum efficiency of the monochromator-detector combination [13] and the absolute Ar 3s-electron photoionization cross sections as a function of the exciting-photon energy [3] are known the absolute $\mathrm{Kr} 4 \mathrm{~s}$-electron photoionization cross section could be determined by comparing the intensities of the Ar and the $\mathrm{Kr}$ lines. Cascade effects have been assumed to be small [2] and were neglected in the present analysis for the $\mathrm{Kr}$ data. In this sense the measured cross sections have to be regarded as an upper limit of the true cross sections.

Figure 2 shows the measured $\mathrm{Kr}$ 4s-electron photoionization cross section as a function of the exciting-photon energy in the range between 30 and 90 $\mathrm{eV}$ together with earlier experimental data $[4,5]$ and calculations $[6,8,14]$. In the low exciting-photon energy region our measured cross sections agree within the uncertainties with the measurements of Samson and Gardner [4]. At higher exciting-photon energies our measured cross sections are systematically smaller than those of Aksela et al. [5]. In ref. [6] an experimental cross section of $0.1 \mathrm{Mb}$ was obtained at

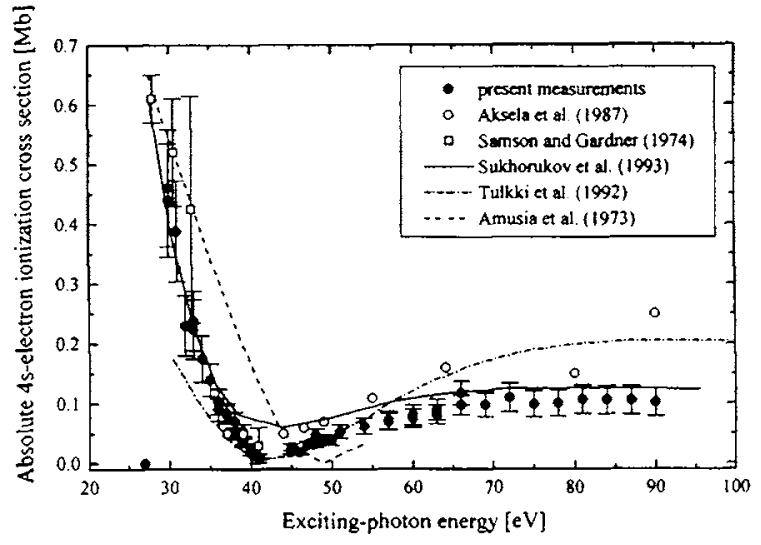

Fig. 2. Absolute KrIl 4s-electron photoionization cross section in the exciting-photon energy range between threshold and $90 \mathrm{eV}$.

$110 \mathrm{eV}$ exciting-photon energy, which is smaller by approximately a factor of two when compared to ref. [5]. This underlines the accuracy of the present measurements. Additionally very recent measurements using PES [7] agree with the present results, too.

The comparison with the various theoretical calculations reveals that the gross features of the measured cross sections as a function of the excitingphoton energy are best described by ref. [8] where the so-called Coulomb interaction correlational decrease [2] and the configuration interaction of the $4 s^{1} 4 p^{62} S_{1 / 2}$-state with the correlation satellites have been included. In the low exciting-photon energy range these new calculations [8] are in good agreement with the experimental cross sections whereas in the high energy range they are somewhat too high but almost within the uncertainties of the present measurements. In the energy range around the minimum the cross sections are best described by ref. [6]. These calculations, however, agree only for energies around the minimum, whereas in other excitingphoton energy ranges they differ considerably from the present measurements. At large photon energies where the calculations [6] exceed our measurements the discrepancies may probably be attributed to the fact that the configuration interaction between the $4 s^{1} 4 p^{6}{ }^{2} S_{1 / 2}$-state and the correlation satellites which would lead to smaller calculated cross sections ( $\mathrm{cf}$. ref. [2]) was neglected in the calculations. The reasons for the calculated cross sections of ref. [6] being 
too small at lower photon energies when compared with the experiment are at present unclear. The very early RPAE calculations [14] seem to be too high in the energy range between 30 and $45 \mathrm{eV}$ and also the exciting-photon energy of the calculated cross section minimum is too high. Both deviations may be related to the neglect of the Coulomb interaction correlational decrease [2] in the calculations [14].

The authors would like to thank Dipl.-Phys. Ralf Henkel for his assistance during the experiments. The continuous help of the staff of BESSY is gratefully acknowledged. This work was funded by the German Federal Minister for Research and Technology under contract nos. 05 SUKAXB and 05 5RGAXB.

\section{References}

[1] V.L. Sukhorukov, B.M. Lagutin, H. Schmoranzer, I.D. Petrov and K.H. Schartner, Phys. Lett. A 169 (1992) 445.

[2] H. Schmoranzer, A. Ehresmann, F. Vollweiler, V.L. Sukhorukov, B.M. Laqutin, I.D. Petrov, K-H. Schartner and B. Mobus, J. Phys. B 26 (1993) 2795.
[3] B. Möbus, B. Magel, K.-H. Schartner, B. Langer, U. Becker, M. Wildberger and H. Schmoranzer, Phys. Rev. A 47 (1993) 3888.

[4] J.A.R. Samson and J.L. Gardner, Phys. Rev. Lett. 33 (1974) 671.

[5] S. Aksela, H. Aksela, M. Levasalmi, K.H. Tan and G.M. Bancroft, Phys. Rev. A 36 (1987) 3449.

[6] J. Tulkki, S. Aksela, H. Aksela, E. Shigemasa, A. Yagishita and Y. Furusawa, Phys. Rev. A 45 (1992) 4640.

[7] B. Langer, private communication (1993).

[8] V.L. Sukhorukov, B.M. Lagutin, I.D. Petrov, H. Schmoranzer, A. Ehresmann and K.-H. Schartner, Photoionization of $\mathrm{Kr}$ near 4s-threshold II. Intermediatecoupling theory, J. Phys. B (1993), in press.

[9] K.-H. Schartner, P. Lenz, B. Möbus, H. Schmoranzer and M. Wildberger, Phys. Lett. A 128 (1988) 374.

[10] K.-H. Schartner, B. Mobbus, P. Lenz, H. Schmoranzer and M. Wildberger, Phys. Rev, Lett. 61 (1988) 2744.

[11] K.-H. Schartner, P. Lenz, B. Mơbus, B. Magel, H. Schmoranzer and M. Wildberger, Phys. Scr. 41 (1990) 853.

[12] H. Schmoranzer, M. Wildberger, K.-H. Schartner, B. Möbus and B. Magel, Phys. Lett. A 150 (1990) 281.

[13] K.-H. Schartner, B. Möbus, M. Wildberger, A. Ehresmann and H. Schmoranzer, BESSY annual report (1991) p. 514.

[14] M.Ya. Amusia, V.K. Ivanov, N.A. Cherepkov and L.V. Chernysheva, abstracts of papers of VIII ICPEAC, eds. B.C. Cobic and M.V. Kurepa (Institute of Physics, Belgrade, 1973) p. 581 . 